\title{
CFD AND METROLOGY IN FLOWMETERING: RCS FLOW MEASUREMENT WITH ELBOW TAPS AND ITS UNCERTAINTY
}

\author{
Olivier Deneux $^{1, \mathrm{a}}$ and Mario Arenas ${ }^{1}$ \\ ${ }^{1}$ EDF R\&D, STEP Department, 6 quai Watier, 78401 Chatou, France
}

\begin{abstract}
For safety reasons, reactor coolant system (RCS) flows in nuclear power plants have to be maintained between a high and a low limit. The current measurement uncertainty is impacted by the heterogeneity of the RCS fluid. The objective of the present study is to use the existing plant elbow taps to measure accurately and absolutely the RCS flow continuously and independently of the temperature measurement. CFD simulations are used to find out a calibration coefficient for the method. An uncertainty calculation is performed. This innovative method was tested on actual data issued from French nuclear power plants.
\end{abstract}

Résumé. Pour des raisons de sûreté, le débit primaire des centrales nucléaires doit rester entre une limite haute et une limite basse. L'incertitude de la mesure actuelle est impactée par l'hétérogénéité de température du fluide primaire.L'objectif de cette étude est d'utiliser les mesures de delta de pression existantes dans certains coudes de centrales nucléaires pour mesurer en absolu et précisément le débit primaire sans que des mesures de température du fluide primaire ne soient nécessaires. La simulation CFD est utilisée pour déterminer un coefficient d'étalonnage de la méthode. Un calcul d'incertitude est alors réalisé. Cette méthode innovante de mesure de débit primaire a été testée sur des données anciennes de centrales nucléaires françaises.

\section{Nomenclature}

CFD Computational Fluid Dynamic

LES Large Eddy Simulation

LLLP Low Leakage Loading Pattern

PWR Pressurized Water Reactor

RANS Reynolds-Averaged Navier-Stokes

RCP114 Periodic test to calculate RCS flow rate

RCS Reactor Coolant System

$\mathrm{V}$ Velocity of the fluid $\left[\mathrm{m} . \mathrm{s}^{-1}\right]$

$\rho$ Density of the fluid $\left[\mathrm{kg} \cdot \mathrm{m}^{-3}\right]$

p Pressure [mbar]

$\mathrm{Q}$ Volume flow rate $\left[\mathrm{m}^{3} . \mathrm{s}^{-1}\right]$

$\mathrm{K}$ Flow coefficient of elbow tap

D Diameter of the pipe [m]

$\mathrm{R}_{\mathrm{c}}$ Radius of curvature of the elbow [m].

$\mathrm{T}$ Temperature of the fluid $\left[{ }^{\circ} \mathrm{C}\right]$

\section{Introduction}

EDF (the French Electricity Generation company) operates 58 PWR-type nuclear units: 34 units of 900MWe 3-loopers, 20 units of 1,300MWe 4-loopers, 4 units of 1,450MWe 4-loopers.

\footnotetext{
${ }^{\mathrm{a}}$ Corresponding author: olivier.deneux@edf.fr
}

Safety is the main concern for plant operation: thus accurate calibration and monitoring of the primary system operation setpoint are of highest importance.

As is well known the RCS (Reactor Coolant System) on the primary side is equipped with limited instrumentation for temperature, flow, and power: thus reliability of these measurements is a key requirement, based on adequate monitoring methods.

Among these parameters, RCS flow (loop flow, and global reactor coolant flow) is to meet specific requirements: RCS flow has to be accurately checked between a high and a low limit (low limit for fuel integrity, and high limit for potential vessel mechanical effects). Currently RCS flow measurement is performed with an indirect method (heat balance between primary and secondary systems), called RCP114 (performed once at the beginning of the cycle at full load): thus accuracy of the measurements of RCS temperatures on hot and cold legs is the main uncertainty contribution.

Because of the measurement technology, uncertainty of the sensors themselves is not the main parameter. Hot leg temperature is measured in a bypass loop fed by extraction of a small flow of the RCS fluid through three 
scoops: it follows that the $\mathrm{T}_{\text {hot }}$ measurement is impacted by this imperfect sampling of the heterogeneous RCS fluid (distribution of hot and cold streams from reactor core). This heterogeneous distribution of hot and cold streams is partially due to the core loading pattern: in recent years this loading pattern has been optimized with particular repartition of the fuel rods in order to limit the neutron flux on the reactor vessel to protect its lifetime. This pattern is called LLLP. Thus emergence of alternative RCS flow measurement technologies independent of the heat balance method may generate interest.

A real-time monitoring system of relative RCS flow exists in order to rapidly detect primary coolant pump failure (rotor breakup or inopportune pump stop). This device uses elbow taps located on each RCS loop between outlet of steam generator and inlet of primary pump. Of course the elbow tap calibration with the heat balance method cannot provide a better accuracy than the heat balance method itself: the study hereafter presented aims at developing and evaluating techniques to investigate possible use of the existing plant elbow taps to accurately and absolutely measure RCS flow, both continuously and independently of the loop temperature measurements.

The original approach mingles both CFD simulation and experimental test on a scale flow bench. CFD is able to predict the elbow tap coefficient with a relevant value (with potential bias as small as possible) and associated accuracy, smaller than that of the heat balance method.

\section{Elbow tap technology for flow}

\subsection{Method description}

Studies and scientific literature already exist about the possibility to measure a flow in a pipe elbow [2] [4]. This method, called elbow tap, is considered as a differential pressure primary device. Indeed the square root of the differential pressure between the flow going along the inner section of the elbow and the flow going along the outer section of the elbow, is in principle proportional to the flow rate. Different factors have an influence on the relation between the square root of the differential pressure and the flow rate.

In order to develop an accurate elbow tap method applied to RCS flow measurement (see the pipe elbow on Figure 1, it is important to identify the most influential factors in the mathematical formula. In order to build this formula, linking the square root of the differential pressure and the flow rate, the flow is assumed to be inviscid (i.e. an ideal fluid with no viscosity). Then the Euler equation over a radial axis can be written:

$$
\rho \frac{V_{\theta}^{2}(r)}{r}=\frac{\partial p}{\partial r}
$$

Figure 1. Elbow tap pressure lines on a RCS pipe in a nuclear power plant loop

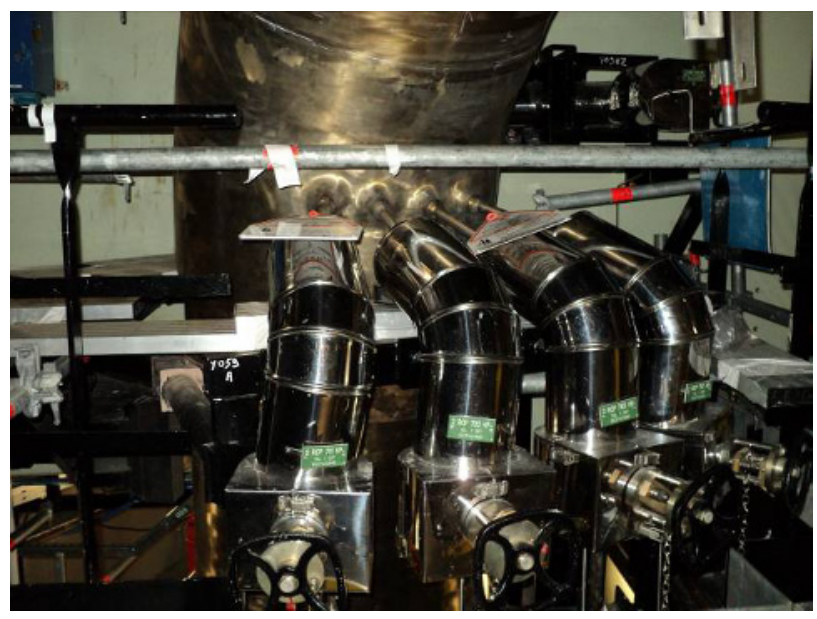

In a first approach, the following basic assumptions are made:

- the velocity of the fluid is uniform over the whole cross section $\left(\mathrm{v}_{\theta}(\mathrm{r})=\mathrm{V}\right)$;

- the diameter of the pipe is much lower than the radius of curvature.

It is therefore possible to integrate the Euler equation over a diameter. This gives the following mathematical equation where both the flow rate and the differential pressure appear:

$$
Q=10 \frac{\Pi}{4} D^{3 / 2} R_{c}^{1 / 2} \sqrt{\frac{\Delta p}{\rho}}
$$

This equation is true in an ideal case. In real conditions, this equation can be approximated with equation (3). The $\mathrm{K}$ coefficient still depends on the flow condition, where $\mathrm{K}$ is close to $10 \Pi / 4$ with a correction factor resulting from the real flow conditions.

$$
Q=K D^{3 / 2} R_{c}^{1 / 2} \sqrt{\frac{\Delta p}{\rho}}
$$

Equation (3) is used by EDF R\&D to develop a new RCS flow measurement method for its PWR fleet. Figuring out the value of this coefficient is the main aim to get an accurate measurement with an elbow tap. EDF would then use its existing taps in the $40^{\circ}$ pipe elbows at the primary outlet of the steam generators in order to measure the differential pressure. In the French 1,450MW PWRs, there is one tap in the outer section of the elbow and four taps in the inner section of the elbow (see Figure 2). 
Figure 2. Pressure taps in the $40^{\circ}$ elbow on a 4-looper PWR

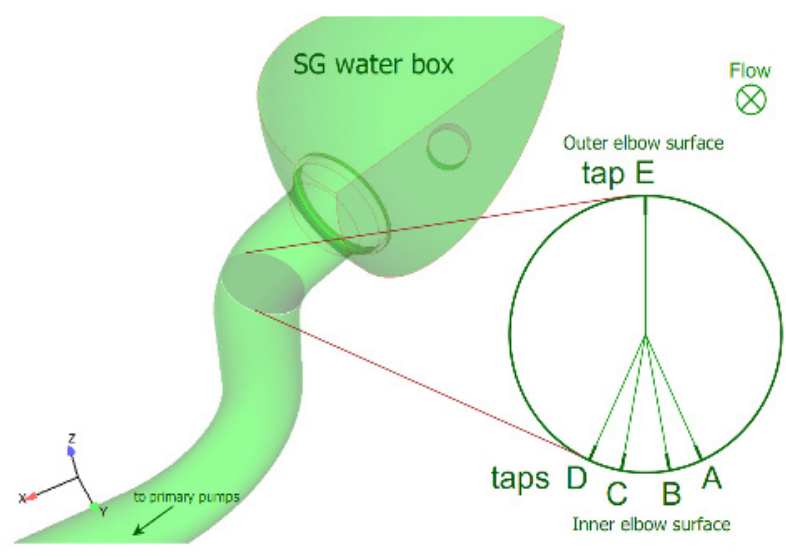

\subsection{Elbow tap for RCS flow measurement}

Equation (3) used by EDF R\&D requires the knowledge of a "K" coefficient. This coefficient can be seen as a calibrating coefficient. Even if some influence factors appear directly in the formula, the $\mathrm{K}$ coefficient may still depend on flow conditions such as the inlet velocity profile of the flow, the fluid temperature, the exact geometry of the pipe elbow, etc. So the K coefficient is not exactly a "universal" constant and may vary if the conditions of the flow change. The aim of the calibration of equation (3) is to figure out a value for this coefficient as relevant as possible for a RCS flow measurement in terms of exactness and uncertainty. EDF R\&D has developed a methodology in order to achieve that.

EDF owns a varied range of data that can help the study of this $\mathrm{K}$ coefficient. Those data come from the following sources:

- measurements from nuclear power plants (EDF owns and operates 58 PWRs, see above);

- experiments on a 1/4 scale model flow test loop, called EVEREST, where the flow rate, the temperature of the fluid and the pressure of the fluid are lower than those of real plant operation condition (see the section concerning the experimentation on a scale model);

- simulation from a CFD code developed by EDF (this open-source code is named Code_Saturne ${ }^{\circledR}$.

The conclusions that can be made from those sources are different. Indeed to compute out the $\mathrm{K}$ coefficient from the nuclear plants measurements, a reference value of the RCS flow rate is required. But the unique current method to get the RCS flow rate value is the RCP114, which is the very method we want to improve. Since the RCS pump curve may evolve during the plant life, the pump $\Delta p$ is not recorded for flow rate measurement. EVEREST is a $1 / 4$ scale model test bench where inlet parameters are lower than real values. Getting conclusions from those experiments only may not be relevant enough. So the methodology developed by EDF $\mathrm{R} \& \mathrm{D}$ to find out the value for the $\mathrm{K}$ coefficient consists in doing some simulations with Code_Saturne ${ }^{\circledR}[1]$. The real measurements on-site and the experiments on EVEREST will be used to validate the method and to carry out the accuracy calculation.

The first step of the methodology consists in creating a pipe computational domain that is adequate enough to represent the RCS flow. The computational domain represents the outlet water box of the steam generator and the $40^{\circ}$ pipe elbow following. It is important to model the water box because its effect on the flow may be important. The computational grid must have at least two millions cells to ensure the mesh convergence and can be conforming or non-conforming. The mesh convergence was studied with different grids containing one million, two millions and four millions cells. The main flow characteristics and the pressure values stop evolving from a two millions cells simulation to a four million cells simulation. Thus two millions cells is enough for this study.

The second step consists in defining the parameters of the simulation. One important choice regards the turbulence model. A LES model is much time consuming and cannot be applied with such a complex flow. So the right choice is to use a RANS model. Different RANS models have been developed such as $\mathrm{k}_{\varepsilon}$ or $\mathrm{k}_{\omega}$, first order models, or $\mathrm{R}_{\mathrm{ij}} \mathrm{SSG}$, second order model. Numerical experiments have been performed from which it seems that the standard $\mathrm{k}_{\varepsilon}$ model is not precise enough for our purpose and that the $\mathrm{R}_{\mathrm{ij}} \mathrm{SSG}$ do not improve that much the results but is more time consuming. So the turbulence model SST $\mathrm{k}_{\omega}$ has been chosen for this study. Finally the inlet values have to be specified such as the velocity of the fluid at the inlet of the water box (supposed to be constant and uniform), the density of the fluid, the viscosity of the fluid, etc. Those values can be evaluated from the operating condition of a nuclear power plant. For a French 1,450MW PWR, those operating conditions are a cold leg temperature of $289^{\circ} \mathrm{C}$, a pressure of 155 bar and a flow rate of $24,840 \mathrm{~m}^{3} \mathrm{~h}^{-1}$.

The third step is then the simulation. The time resolution of the simulation has to be quite small, $10^{-2} \mathrm{~s}$ in this study. The simulation is well converged after 2,000 to 3,000 iterations. A picture of the global geometry of the pipe and the simulation can be seen on Figure 3

The fourth and final step is the post-processing of the results. The CFD gives the pressure field in the whole domain. Thus it is now possible to get the pressure in the different tap locations. Then a $\mathrm{K}$ coefficient can be computed using equation (4) since the last unknown was the differential pressure (the flow rate being an inlet parameter of the simulation).

$$
K=\frac{Q}{D^{3 / 2} R_{c}^{1 / 2} \sqrt{\frac{\Delta p}{\rho}}}
$$


Figure 3. CFD of the RCS flow

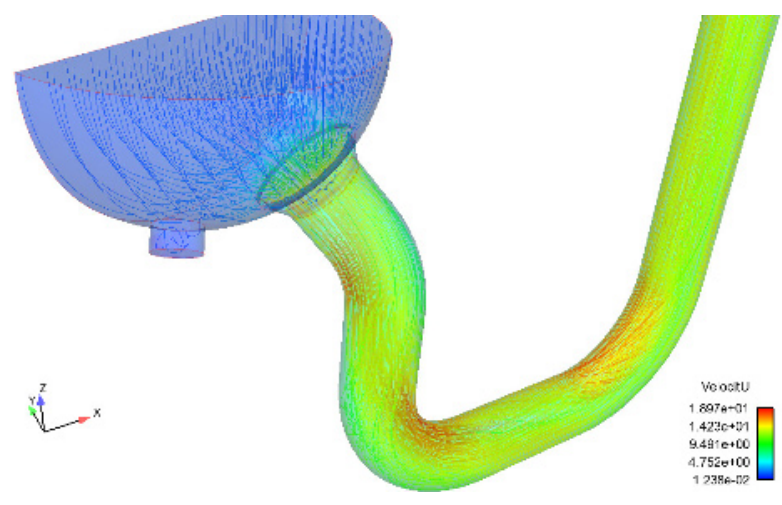

Eventually four K coefficients are computed from the four differential pressures: one for each tap of the inner surface. The four differential pressures are directly taken from the CFD results as seen in the Figure 4. A patent has protected this methodology since July 2011 [3]. Since measuring a flow rate from an elbow tap is not innovative, the patent is based on the calibrating process, using CFD, for measuring an industrial flow rate with high accuracy.

Figure 4. Pressure and velocity profiles in the nozzles' section

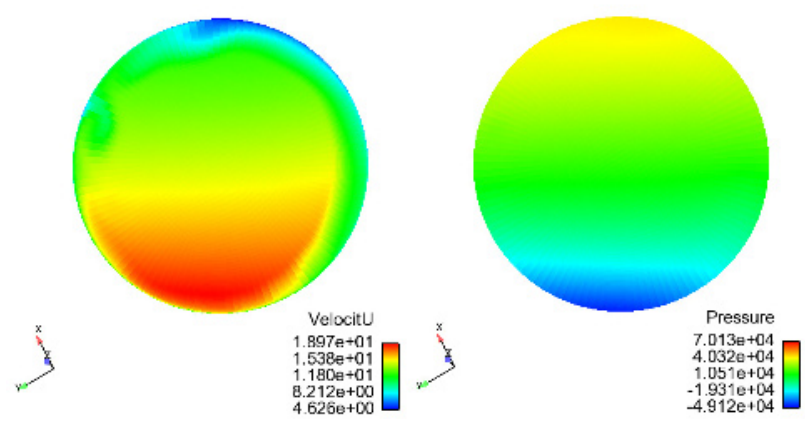

\section{Uncertainty quantification of $K$ coefficient}

\subsection{Method description}

As described in a large number of publications, the uncertainty quantification in CFD is based on identification and estimation of the effects of all sources of uncertainty. A well accepted technique is the V\&V [5] method that defines Verification as the activity to evaluate the numerical error, and Validation as the activity to evaluate physical uncertainties. Inspired on $\mathrm{V} \& \mathrm{~V}$, EDF's method consists of the identification and characterization of each source of uncertainty. Then different groups are defined and a method for its estimation is applied. The choice of a realistic method for each uncertainty source is based on available data at EDF: actual measurements from PWRs and experimental data on a $1 / 4$ scale model flow test loop. Some sources have been identified but neglected because not significant, and the others identified and estimated with a conservatism approach for safety reasons. The last step consists of estimating the total uncertainty of the $\mathrm{K}$ coefficient. Combined uncertainty is calculated with conservative hypotheses. The quadratic summation also known as 'root sum of the squares' is reasonable in this instance as described further.

\subsection{Characterization and estimation of uncertainty sources}

Identification step provides a list of uncertainty sources:

$$
\begin{aligned}
& \text { - physical model; } \\
& \text { - time and space discretization; } \\
& \text { - input data; } \\
& \text { - iterative convergence; } \\
& \text { - round-off; } \\
& \text { - computer programming. }
\end{aligned}
$$

From this large but not exhaustive list, the physical model and the space discretization source are evaluated as a unique source. First, because all of them have a common origin: simplifications from real phenomenon. Turbulence model, boundary conditions, physical properties and geometry are considered on this group. On the other hand no affordable method exists (in terms of time calculation). Thus estimation of uncertainty is made by $1 / 4$ scale model data comparison. EDF R\&D operates its own flow test loop (with a $\pm 0.4 \%$ flowmeter) where a $1 / 4$ scale model is installed (see Figure 5).

Figure 5. 1/4 scale mock-up in flow test loop EVEREST

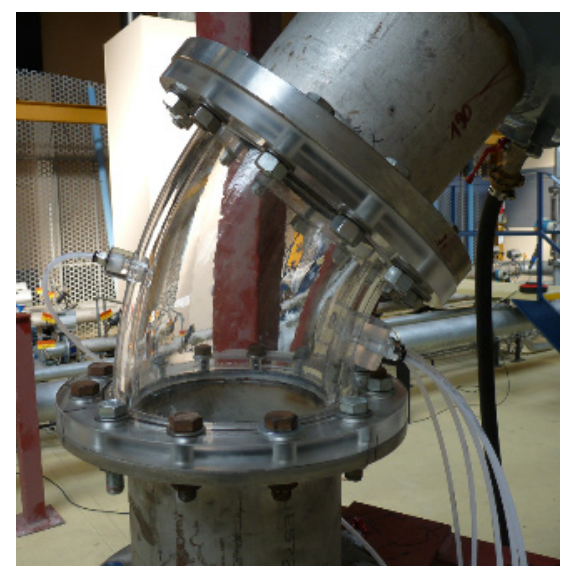

Using equation (4), an experimental $\mathrm{K}$ coefficient can be calculated accurately. Then the mean deviation between experimental and CFD $\mathrm{K}$ coefficients are calculated for various flow rates. A normal (or Gaussian) distribution is chosen to cover amply $\mathrm{K}$ coefficient deviation data. For extrapolating from $1 / 4$ scale to full scale model an analysis based on a dynamic similitude law is made. For the same Reynolds number $\left(2.10^{6}\right)$ two simulations are made, the first one at $1 / 4$ scale conditions (temperature of $26^{\circ} \mathrm{C}$ and $\mathrm{D}=0.2 \mathrm{~m}$ ) and the second one at full scale conditions (temperature of $289{ }^{\circ} \mathrm{C}$ and 
$\mathrm{D}=0.78 \mathrm{~m})$. Results show that $\mathrm{K}$ coefficients are comparable. The second part of this analysis is the evolution of $\mathrm{K}$ coefficient vs Reynolds number for scaled and full model (see Figure 6). $\mathrm{K}$ coefficient is quite stable and no important dependencies exist.

Figure 6. K coefficient vs. Reynolds number

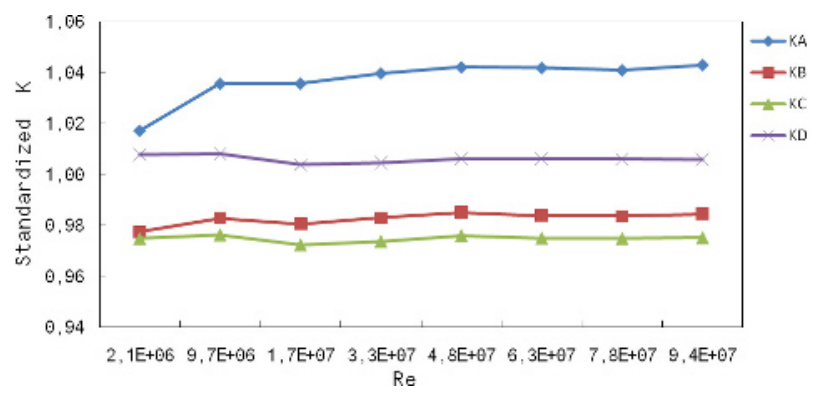

Regarding input data, EDF R\&D evaluates its influence by a sensitivity test of the velocity and the temperature inputs. Taking advantage of actual measurements from PWR, three levels for CFD input data are chosen. First a nominal value $\left(T_{\text {nom }}\right.$ and $\left.V_{\text {nom }}\right)$ is defined based on nominal operating conditions. Then minimum and maximum values are defined from safety limits. Finally CFD simulations are executed for different combinations of levels $\left(\mathrm{T}_{\min } / \mathrm{V}_{\max }, \mathrm{T}_{\max } / \mathrm{V}_{\min }\right.$, etc.). Results are analyzed by a statistic approach. A mean value and a standard deviation are calculated based on a normal distribution (coverage factor of $2 \sigma$ ).

As said above, $\mathrm{K}$ coefficient is computed using equation (4) where the differential pressure is taken from simulations results. $\mathrm{K}$ coefficient varies lightly as a function of sampling number used for calculation. Analyzing all CFD results permits to choose an optimal sampling number. Variations of differential pressure are computed for the last 500 iterations where convergence is guaranteed. Once more an average and standard deviation is calculated from the last 500 iterations via normal distribution method. For round-off and computer programming uncertainty sources are insignificant and not considered in this study.

Uncertainty estimation as shown above deals only with differential pressure calculation from equation (4) due to CFD errors. Thermal expansion might also have an effect on $\mathrm{K}$ coefficient uncertainty due to temperature difference, from $25^{\circ} \mathrm{C}$ to $289^{\circ} \mathrm{C}$. Based on well-know coefficient of thermal expansion for stainless steel, an estimation is done for $\mathrm{K}$ coefficient for low temperature and nominal operating temperature. Results show the effect is small but not negligible: deviation reaches $1 \%$.

\subsection{Estimation of uncertainty budget: summation with quadratic method}

Physical model and discretization uncertainty represent systematic error that might be corrected when reference values are available. In this case that's not possible because the bias is known only for scaled model. Input data uncertainty represents a random error that must be related to physical model and discretization uncertainty. Those uncertainty sources are not independent and covariances must also be taken into account when calculating the combined uncertainty.

Uncertainties due to iterative convergence and thermal expansion are mutually independents and can be combinated by quadratic summation method. Uncertainty budget is finally expressed as:

$$
\sigma_{\text {total }}=\sqrt{\left(\sigma_{\text {inp }}+\varepsilon_{\text {mod }}\right)^{2}+\sigma_{c o n v}{ }^{2}+\sigma_{\text {ther }}^{2}}
$$

where:

$\sigma_{\text {total }}$ uncertainty budget of K coefficient;

$\sigma_{\text {inp }}$ uncertainty of inputs data;

$\varepsilon_{\text {mod }}$ uncertainty of physical model and space discretization;

$\sigma_{\text {conv }}$ uncertainty due to iterative convergence;

$\sigma_{\text {ther }}$ uncertainty due to thermal expansion.

\section{Conclusion}

The current method to calculate RCS flow rate in nuclear power plants can be influenced by the temperature heterogeneity in the hot leg of the primary loop. A method using the existing elbow taps at the outlet of the steam generator should be able to solve this issue. EDF R\&D has developed a new method in order to get a continuous, accurate and absolute measurement of the RCS flow rates, that is independent of the loop temperature measurements.

Once known, the uncertainty of the $\mathrm{K}$ coefficient, from the equation (3), can be used to calculate the global uncertainty of the flow rate measurement. Due to the redundancy of the differential pressures on PWRs, the global uncertainty of the method is below 3\%. Thus this RCS flow measurement is more accurate than the RCP114.

Figure 7. Comparisons between the RCP114 and differential pressure flow rates in the Civaux unit 1

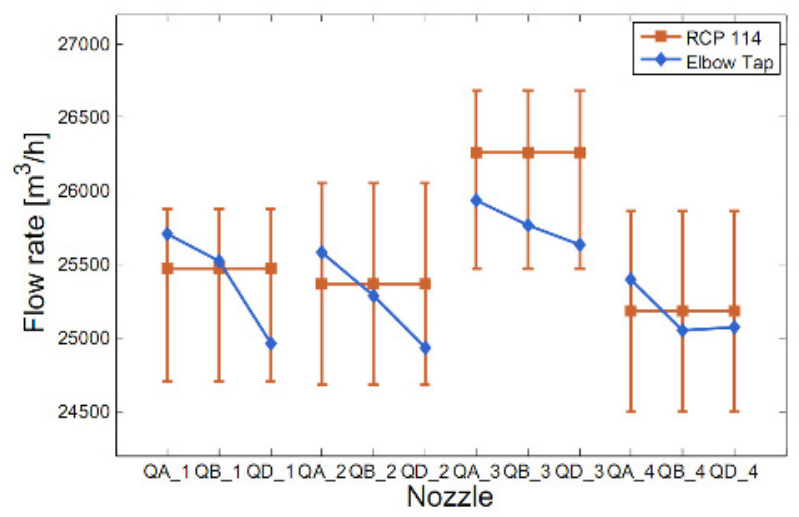


Real tests were performed on the Civaux plant (two 4looper 1,450MW units). Three flow rates resulting from three differential pressures have been calculated for each loop (see Figure 7). All measurements are coherent with the RCP114 flow rate. This can be considered as a first validation of the method.

\section{Acknowledgements}

This study was a collaborative work involving various EDF teams: the authors are grateful for the help and contribution of both STEP department (scale model experiments and uncertainty method) and MFEE departement (CFD expertise) from the EDF R\&D as well as nuclear operators who provided essential plant data.

\section{References}

[1] F. Archambeau, N. Méchitoua, and M. Sakiz. Code_Saturne: a Finite Volume Code for the Computation of Turbulent Incompressible Flows. International Journal on Finite Volumes, Vol. 1, 2004.

[2] M. A. Crabtree. Industrial Flow Measurement. Master's thesis, University of Huddersfield, 2009.

[3] O. Deneux, J. M. Favennec, and J. Veau. Suivi de débit dans une conduite hydraulique fermée, 07 2011.

[4] R.W. Miller. Flow Measurement Engineering Hanbook. 1976.

[5] W.L. Oberkampf, T.G. Trucano. Validation Methodology in Computational Fluid Dynamics. Sandia National Laboratories. 2000. 